Errard, C. 1984.

[Oct.1984]

Insectes Sociaux, Paris

1984, Volume $31, n^{\circ} 2$, pp. $185-198$

(C) Masson, Paris, 1984

\title{
EVOLUTION, EN FONCTION DE L'AGE, DES RELATIONS SOCIALES DANS LES COLONIES MIXTES HÉTÉROSPÉCIFIQUES CHEZ LES FOURMIS DES GENRES CAMPONOTUS ET PSEUDOMYRMEX *
}

\author{
C. ERRARD \\ Equipe de Recherche Associée au C.N.R.S. $n^{\circ} 885$ \\ Laboratoire d'Ethologie et Sociobiologie, Université Paris XIII, F 93430 Villetaneuse \\ et C.I.E.S., Tapachula, Chiapas, Mexique
}

Reçu le 21 juin 1983.

Accepté le 10 février 1984.

\section{RESUME}

Dans les colonies mixtes réalisées (Camponotus senex - Camponotus abdominalis, Camponotus senex - Camponotus species, Camponotus senex - Pseudomyrmex ferruginea) on confirme que la probabilité d'obtenir une société mixte à partir des adultes est d'autant plus grande que les individus utilisés sont plus jeunes et que la distance phylogénétique entre les espèces en présence est plus faible.

L'intégration sociale a été évaluée en notant d'une part la position des fourmis dans le nid et d'autre part la tolérance entre les espèces, sur la source de nourriture (approvisionnement).

Les relations homospécifiques et hétérospécifiques sont quantifiées en relevant la fréquence des comportements ayant un rapport direct avec la distribution de nourriture, le transport du couvain et les toilettes.

A l'intérieur du genre Camponotus, l'association serait du type symbiotique alors que dans une colonie mixte associant deux sous-familles (Camponotus senex - Pseudomyrmex ferruginea), elle ressemblerait plutôt à un parasitísme social.

\section{SUMMARY}

\section{Study of the social relationships In \\ hetero-specific mixed colonies of ants as a function of age}

In the artificial mixed colonies (Camponotus senex - Camponotus abdominalis. Camponotus senex - Camponotus species, Camponotus senex - Pseudomyrmex ferruginea),

* Recherche réalisée avec l'aide du Ministère de l'Education Nationale ("Aide à la Recherche Universitaire, Biologie, 1983 »). 
the results obtained before have been confirmed with other species: the probability to obtain a mixed society from adults is all the more high as the animals used are younger and the phylogenetic distance between the species faced each other is smaller.

The social integration was evaluated by noting on the one hand the position of the ants in the nest and on the other the tolerance between individuals of the two species, at the food source (foraging).

The homo-specific and hetero-specific relationships are quantified by noting the frequencies of the behaviours which have a direct connection with the trophallactic behaviour, brood-carrying and grooming.

For the Camponotus species, the association would be of the symbiotic type as well as it would be like a social parasitism in a mixed colony which connects two subfamilies (Camponotus senex - Pseudomyrmex ferruginea).

\section{INTRODUCTION}

Dans la nature, des fourmis d'espèces différentes peuvent s'associer pour constituer des fourmilières mixtes. Ces associations ont été qualifiées, soit de " parasitisme social » soit de "symbiose sociale ". STUMPER (1950) y distingue les cas de deux (ou plusieurs) espèces de fourmis vivant régulièrement ou obligatoirement en commun, (c'est-à-dire où l'une des espèces est assimilée à l'autre), de ceux où l'association est moins intime. Il existe de nombreux exemples répartis dans différentes sous-familles (ForEL, 1898; MANN, 1912 , WHEELER, 1926 ; KUTTER, 1945).

FIELDE (1903) a constitué des sociétés mixtes artificielles à l'aide d'espèces ne pratiquant jamais ce type d'association. Depuis, les sociétés mixtes artificielles de fourmis adultes ont été peu étudiées alors que celles associant adultes et larves furent plus souvent observées (HeYde, 1924 ; Plateaux, 1960 a et $b$; Benors, 1969).

Ce modèle expérimental des sociétés mixtes a été repris par JaIsson (1980), qui émit l'hypothèse que la "probabilité d'obtenir une société mixte est d'autant plus grande que les individus utilisés sont plus jeunes et que la distance phylogénétique entre espèces est plus faible ».

Nous nous sommes proposé d'étudier ici certains aspects éthologiques de l'intégration sociale dans ces sociétés. Cette intégration sociale est analysée en fonction de l'âge des individus au moment de la confrontation et de la distance phylogénétique. Nous avons utilisé des sous-familles fort différentes : les Formicinae, venant des formicoïdes primitifs et les Pseudomyrmecinae, issus des poneroïdes primitifs (TAYLOR, 1978).

Nous avons porté notre étude sur la tolérance transitoire durant laquelle les stimulations hétérospécifiques n'induisent pas l'agression, et à étudier la variabilité de la plasticité comportementale en fonction de l'âge des individus. 


\section{MATERIEL ET METHODES}

Parmi les Formicinæ, nous avons étudié Camponotus senex (dont les nids tissés rappellent les cocophylles), Camponotus abdominalis (terricoles) et Camponotus sp. (nid dans le bois mort). Une seule espèce de Pseudomyrmecina a été utilisée: Pseudomyrmex ferruginea (myrmécophyte de l'acacia). Le choix des espèces est basé essentiellement sur leur abondance dans la région de Tapachula (Sud-Est du Mexique) où nous les avons récoltées en août et septembre 1982.

Les expériences furent réalisées à $32^{\circ} \mathrm{C} \pm 3^{\circ} \mathrm{C}$, photopériode $12 / 12,70 \%$ H.R. ; alimentation avec miel et larves de termites. Chaque colonie mixte est élevée dans un nid en tube de verre de $18 \mathrm{~cm}$ de long et $1,7 \mathrm{~cm}$ de diamètre, avec réserve d'eau. La nourriture est déposée du côté sec, exposé à la lumière.

Une colonie mixte de deux espèces est formée de 10 jeunes ouvrières de chaque introduites dans le tube avec cinq nymphes ou cocons de leur couvain respectif. Les ouvrières, rigoureusement du même âge, sont rassemblées dans le tube soit dès l'émergence, soit après une période d'élevage en groupes homospécifiques variant de 1 a 7 jours. Des colonies témoins ont été réalisées, avec 20 ouvrières d'une seule espèce rassemblées dès l'émergence avec 10 nymphes ou cocons de leur couvain. Deux modalités d'observation ont été utilisées:

- directement dans le tube, 1 heure par jour pendant 15 à 20 jours, afin de relever les comportements en rapport direct avec l'approvisionnement, la distribution de nourriture, le transport du couvain, les toilettes interindividuelles;

- à l'aide d'enregistrements photographiques donnant la répartition des individus.

Ces modalités d'observation nous ont permis d'atteindre une évaluation du degré de cohabitation ainsi que des relations interindividuelles.

\section{1) Evaluation de la cohabitation}

a) Dans nos expériences, les colonies ne sont alimentées que lors de l'observation. Celle-ci consiste à noter, toutes les minutes, les comportements d'approvisionnement qui succèdent à l'introduction de nourriture puis évaluer le niveau de recouvrement spatial et temporel de l'espace vital.

Un indice $I$, dit de "tolérance » sur la source de nourriture, a été défini ainsi :

$$
I=\frac{\Sigma a+b}{\Sigma a^{\prime}+\Sigma b^{\prime}+\Sigma a+b}
$$

où $\Sigma a+b$ représente la somme des individus de chaque espèce $(A+B)$ s'approvisionnant simultanément.

$\Sigma a^{\prime}=$ somme des individus de l'espèce A s'approvisionnant seuls.

$\Sigma \mathbf{b}^{\prime}=$ somme des individus de l'espèce B s'approvisionnant seuls.

Cet indice tend vers 0 lorsque les rapports entre l'espèce $A$ et l'espèce $B$ sont conflictuels et vers 1 lorsque l'interpénétration des deux espèces est totale.

b) En dehors des périodes d'observation directe, la répartition des individus a été notée grâce aux enregistrements photographiques.

Cette analyse permet de déterminer l'occupation de l'espace qui a été traduite sur une échelle qui donne le degré de cohésion sociale depuis le partage de l'espace jusqu'à son utilisation de façon homogène par les deux espèces : 0 , aucune cohésion entre les espèces; + , juxtaposition des deux espèces $;++$, cohésion partielle $;+++$, cohésion totale. 


\section{2) Relations interindividuelles}

Ces relations sont analysées en quantifiant les échanges trophallactiques, les toilettes interindividuelles, le transport du couvain. Ce dernier est induit par une légère perturbation entraînant la dispersion du couvain dans tout le tube (celui-ci est reconnu par sa forme, sa taille ou par une tache de couleur).

Les expériences ont été réalisées sur 2 colonies tests (minimum) pour chaque type d'association; la variabilité d'une colonie à l'autre étant faible, nos résultats portent sur la moyenne des 2 ou $n$ colonies.

\section{RESULTATS}

\section{Camponotus senex - Camponotus abdominalis}

Selon les cas, les individus ont été réunis dès l'émergence ou le $4^{\circ}, 5^{\circ}, 6^{\circ}$ ou $7^{\circ}$ jour après. Lorsque les ouvrières sont âgées de 7 jours lors de la confrontation, les comportements agressifs entraînent rapidement un taux de mortalité de $100 \%$, empêchant la réalisation des tests.

\section{Cohabitation}

Lorsque les individus sont placés en colonie mixte dès l'émergence, les contacts sont immédiats et la répartition s'uniformise dès le premier jour. Par contre, la population est nettement divisée lorsque les individus sont réunis le $4^{\circ}, 5^{\circ}$ ou $6^{\circ}$ jour après l'émergence; la cohésion sociale ne s'établit alors que 4 ou 5 jours après (tableau I).

Le tableau II montre que la tolérance sur la source de nourriture s'installe progressivement et diminue au fur et à mesure que l'âge de mise en présence des individus augmente. Aindi, l'indice de tolérance passe, de 0,65 quand les animaux sont réunis dès l'émergence, à 0 quand ils le sont à l'âge de 6 jours.

L'étude détaillée montre que la proportion de $C$. senex pourvoyeuse augmente progressivement (de $51 \%$ à $89 \%$ ) en fonction de leur âge lors de la confrontation, tandis qu'elle diminue corrélativement chez $C$. abdominalis, (de $49 \%$ à $11 \%$ ), $\chi^{2}=17,25, p<0,001$ ). Il apparaît que les $C$. senex s'approvisionnent une fois et demie plus que dans la colonie témoin, subissant ainsi une légère stimulation en présence des $C$. abdominalis.

\section{Relations interindividuelles}

Les résultats (fig. 1 a) montrent qu'en fonction de l'âge de mise en présence, la proportion des échanges trophallactiques homospécifiques augmente régulièrement (de $20 \%$ à $91 \%$ ) chez $C$. senex, alors qu'elle diminue (de $40 \%$ à 0$)$ chez $C$. abdominalis, $\left(\chi^{2}=94,29, \mathrm{p}<0,001\right)$. Lors des échanges trophallactiques hétérospécifiques, les $C$. senex reçoivent plus qu'ils ne donnent, cette tendance diminuant cependant avec l'âge de mise en présence (32\% à l'émergence, $5 \%$ le $6^{\circ}$ jour $),\left(\chi^{2}=15,33, \mathrm{p}<0,005\right)$. La durée des trophallaxies varie avec la nature des espèces. La durée des échanges homospécifiques est 
Tableau I. - Estimation de la cohésion sociale des 2 espèces en fonction de la durée de l'expérience et de l'âge des ouvrières lors de la mise en présence:

C. senex - C. abdominalis;

C. senex - Camponotus sp.;

$C$. senex - $P$. ferruginea.

$0 \quad$ : aucune cohésion.

$+\quad:$ juxtaposition des espèces.

$++:$ cohésion faible.

$++4:$ cohésion totale.

Table I. - Evaluation of the social cohesion as a function of the age of both species when they are faced:

C. senex - C. abdominalis;

C. senex - Camponotus sp.;

C. senex - $P$. ferruginea.

0 : no cohesion.

$+\quad$ : low cohesion.

++ : large cohesion.

+++ : complete cohesion.

\begin{tabular}{|c|c|c|c|c|c|c|c|}
\hline \multirow[t]{2}{*}{ Espèces } & \multirow{2}{*}{$\begin{array}{l}\text { Age des } \Varangle \\
\text { à la mise } \\
\text { en présence }\end{array}$} & \multicolumn{6}{|c|}{ Durée de l'expérience } \\
\hline & & $0 \mathrm{j}$ & $1 \mathrm{j}$. & $2 \mathrm{j}$ & $3 \mathbf{j}$ & $4 \mathrm{j}$. & $5 \mathrm{j}$. \\
\hline \multirow{5}{*}{$\begin{array}{l}\text { Camponotus } \\
\text { senex - } \\
\text { Camponotus } \\
\text { abdominalis }\end{array}$} & Emergence & ++ & +++ & +++ & +++ & +++ & +++ \\
\hline & 4 jours & 0 & 0 & + & + & ++ & +++ \\
\hline & 5 jours & 0 & 0 & 0 & + & + & +++ \\
\hline & 6 jours & 0 & 0 & 0 & 0 & + & $++t$ \\
\hline & 7 jours & \multicolumn{6}{|c|}{ Mortalité : $100 \%$} \\
\hline \multirow{5}{*}{$\begin{array}{l}\text { Camponotus } \\
\text { senex- } \\
\text { Camponotus } \\
\text { species }\end{array}$} & Emergence & 0 & + & +++ & +++ & +++ & +++ \\
\hline & 1 jour & 0 & + & ++ & ++ & ++ & $++t$ \\
\hline & 3 jours & 0 & 0 & +++ & +++ & +++ & $++t$ \\
\hline & 4 jours & 0 & 0 & + & +++ & +++ & $++t$ \\
\hline & 6 jours & \multicolumn{6}{|c|}{ Mortalité : $100 \%$} \\
\hline \multirow{3}{*}{$\begin{array}{l}\text { C. senex - } \\
\text { Pseudomyrmex } \\
\text { ferruginea }\end{array}$} & Emergence & + & +++ & +++ & +++ & +++ & $+t+$ \\
\hline & 1 jour & 0 & + & +++ & $+t+$ & +++ & $++t$ \\
\hline & 2 jours & \multicolumn{6}{|c|}{ Mortalité : $100 \%$} \\
\hline
\end{tabular}

moyenne ( $>30 \mathrm{~s}$ et $<60 \mathrm{~s})$ dans $90 \%$ des cas et longues $(>60 \mathrm{~s})$ dans $7 \%$. Par contre, celle des échanges hétérospécifiques est soit moyenne $(80 \%)$ soit longue $(3 \%)$ soit courte $(<30 \mathrm{~s}, 17 \%)$.

La figure $1 \mathrm{~b}$ montre que la proportion du transport du couvain homospécifique varie en fonction de l'âge de mise en présence des individus : elle augmente de 31 à $66 \%$ chez $C$. senex alors qu'elle passe de $32 \%$ à $17 \%$ chez C. abdominalis, $\left(\chi^{2}=4,87\right.$, N.S.). 
Tableau II. - Indice de tolérance entre les 2 espèces sur la source de nourriture en fonction de la durée de l'expérience et de l'âge des ouvrières lors de la mise en présence :

C. senex - C. abdominalis ;

C. senex - Camponotus sp.;

C. senex - P. ferruginea.

Table II. - Tolerance index about the source of food as a function of the experiment duration :

C. senex - C. abdominalis ;

C. senex - Camponotus sp.;

C. senex - $P$. ferruginea.

\begin{tabular}{|c|c|c|c|c|c|}
\hline \multirow[t]{2}{*}{ Espèces } & \multirow{2}{*}{$\begin{array}{c}\text { Age } \\
\text { des Camponotus } \\
\text { à la mise } \\
\text { en présence }\end{array}$} & \multicolumn{4}{|c|}{ Durée de l'expérience } \\
\hline & & $0.4 \mathrm{j}$ & $5.9 \mathrm{j}$ & $10-14 \mathrm{j}$ & 1 moyen \\
\hline \multirow{4}{*}{$\begin{array}{l}\text { Camponotus } \\
\text { senex - } \\
\text { Camponotus } \\
\text { abdominalis }\end{array}$} & Emergence & 0,41 & 0,67 & 0,85 & 0,65 \\
\hline & 4 jours & 0,27 & 0,81 & 0,73 & 0,61 \\
\hline & 5 jours & 0,05 & 0,48 & 0,57 & 0,37 \\
\hline & 6 jours & 0 & 0 & 0 & 0 \\
\hline \multirow{4}{*}{$\begin{array}{l}\text { Camponotus } \\
\text { senex- } \\
\text { Camponotus } \\
\text { species }\end{array}$} & Emergence & 0,67 & 0,55 & 0,57 & 0,60 \\
\hline & 1 jour & 0,44 & 0,55 & 0,57 & 0,52 \\
\hline & 3 jours & 0,38 & 0,41 & 0,50 & 0,45 \\
\hline & 4 jours & 0,23 & 0,30 & 0,29 & 0,28 \\
\hline \multirow{2}{*}{$\begin{array}{l}\text { C. senex - } \\
P . \text { ferruginea }\end{array}$} & Emergence & 0,62 & 0,80 & 0,70 & 0,68 \\
\hline & 1 jour & 0,57 & 0,58 & 0,83 & 0,66 \\
\hline
\end{tabular}

Quant au transport hétérospécifique, il est assuré en grande partie (28\%) par $C$. abdominalis quand les individus sont réunis à l'émergence mais il est repris par $C$. senex $(29 \%)$ lorsque les jeunes sont réunis à l'âge de 4 ou 5 jours, $\left(x^{2}=32,54, p<0,001\right)$.

Nous observons des variations semblables pour les toilettes interindividuelles (fig. $1 \mathrm{c}$ ) : les toilettes homospécifiques augmentent avec l'âge de mise en présence chez $C$. senex ( $5 \%$ à l'émergence et $54 \%$ le $6^{\circ}$ jour) tandis qu'elles diminuent de $48 \%$ à $12 \%$ chez $C$. abdominalis $(p<0,001)$. De plus, ces derniers sont très souvent initiateurs des toilettes hétérospécifiques, cette activité diminuant également avec l'âge de confrontation ( $38 \%$ à l'émergence et $24 \%$ le $6^{\circ}$ jour $)$, $(\mathrm{p}<0,005)$.

En général (surtout lorsque les individus sont rassemblés le jour de l'émergence), les $C$. abdominalis ont un rôle plus actif que les $C$. senex. Ces derniers s'assurent cependant d'importantes réserves sans en faire bénéficier 

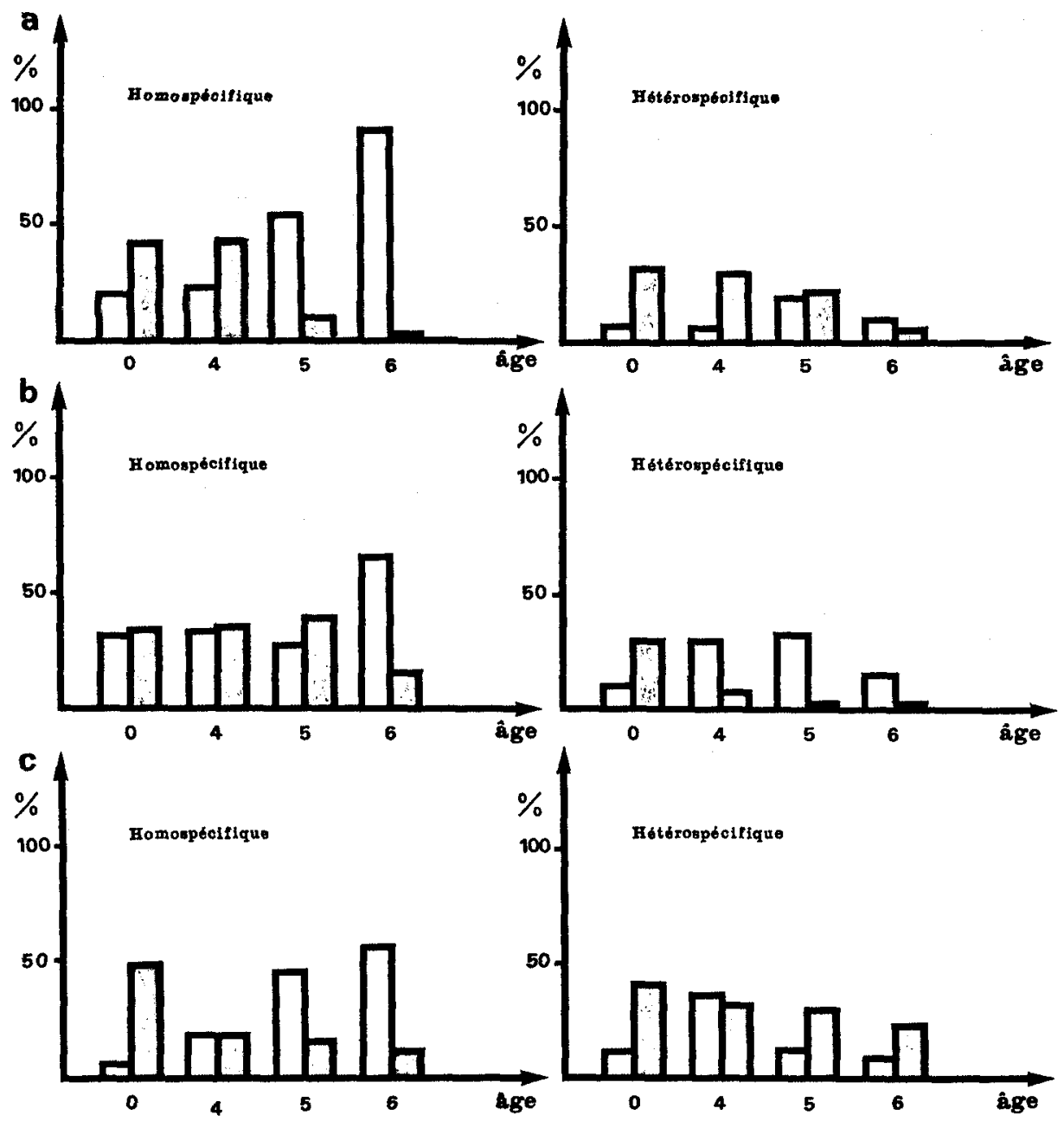

Fig. 1. - Nombre de comportements homo et hétérospécifiques sur le nombre total des comportements, en fonction de l'âge des ouvrières lors de la mise en présence; a, trophallaxie; b, transport du couvain; $c$, toilette.

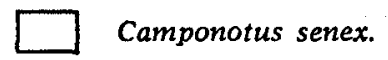

\section{Camponotus abdominalis.}

Fig. 1. - The number of homospecific and heterospecific behaviours of the total number of behaviours as a function of the age of the species when they are faced; a, trophallactic behaviour; b, brood-carrying; $c$, grooming. 
les $C$. abdominalis. Dans cette association, il semblerait donc que $C$. senex vive aux dépens de $C$. abdominalis.

\section{Camponotus senex - Camponotus species}

Cette association a été réalisée avec des individus réunis dès l'émergence, ou le $1^{\text {*r }}, 3^{\circ}, 4^{\circ}$ et $6^{\circ}$ jours. Les tentatives d'association se soldent par un échec à partir du $6^{\circ}$ jour, les agressions aboutissant à la mort rapide des ouvrières.

\section{Cohabitation}

L'intégration des deux espèces est d'autant plus rapide que les individus sont rassemblés plus jeunes (tableau I) : elles se mêlent dès le premier jour lorsque les individus sont réunis à l'émergence, mais seulement le $2^{\circ}$ jour lorsqu'ils sont mis en présence à l'âge de 4 jours.

D'autre part, la tolérance interspécifique sur la source de nourriture diminue régulièrement (de 0,60 à 0,28 ) avec le groupement plus tardif des ouvrières (tableau II), mais il n'apparaît pas de variation nette en fonction de la durée de l'expérience.

Dans cette association, l'essentiel de l'approvisionnement est assuré par Camponotus sp. $\left(68 \%\right.$ contre $32 \%$ chez $C$. senex, $\chi^{2}=5,60$, N.S.) et, pour les deux espèces, ce comportement est semblable à celui observé dans les colonies témoins.

\section{Relations interindividuelles}

La figure 2 a montre que les Camponotus sp. sont donneurs et assurent l'essentiel des échanges trophallactiques, qu'ils soient homospécifiques $(58,5 \%$ contre $12,5 \%$ assurés par $C$. senex, $\chi^{2}=23,10, p<0,001$ ), ou hétérospécifiques $\left(18,5 \%\right.$ contre $10,5 \%$ chez $C$. senex, $\left.\chi^{2}=8,24, p<0,05\right)$.

Les échanges homospécifiques ont une durée moyenne (entre 30 et $60 \mathrm{~s}$ ) dans $95 \%$ des cas, courte $(<30 \mathrm{~s})$ dans seulement $2 \%$, et longue $(>60 \mathrm{~s})$ dans $3 \%$. Par contre, les trophallaxies hétérospécifiques ont une durée moyenne dans $85 \%$ des cas, courte dans $10 \%$, et longue dans $5 \%$.

Ce sont également les Camponotus sp. qui effectuent la plus grande partie des transports de couvain : le transport du couvain homospécifique est d'autant plus important que l'âge de mise en présence des individus est plus élevé (il passe de $37 \%$ à $68,5 \%),\left(\chi^{2}=24,82, \mathrm{p}<0,001\right)$, mais par contre, le transport du couvain hétérospécifique devient nul dès que les fourmis sont réunies à l'âge de 3 et 4 jours $\left(\chi^{2}\right.$, N.S.) (fig. 2 b).

Bien que chez $C$. senex on note une augmentation très importante des toilettes homospécifiques avec l'âge de confrontation des individus (il passe de $3,5 \%$ à l'émergence, à $27 \%$ le $4^{*}$ jour $\left(\chi^{2}=10,54, \mathrm{p}<0,02\right.$ (fig. $\left.2 \mathrm{c}\right)$; les toilettes interindividuelles sont principalement assurées par Camponotus sp. (44 $\%$ contre $3,5 \%$ pour $C$. senex), ( $\chi^{2}$, N.S.).

Dans ce type de colonie, les Camponotus sp., ont l'initiative de la plus 

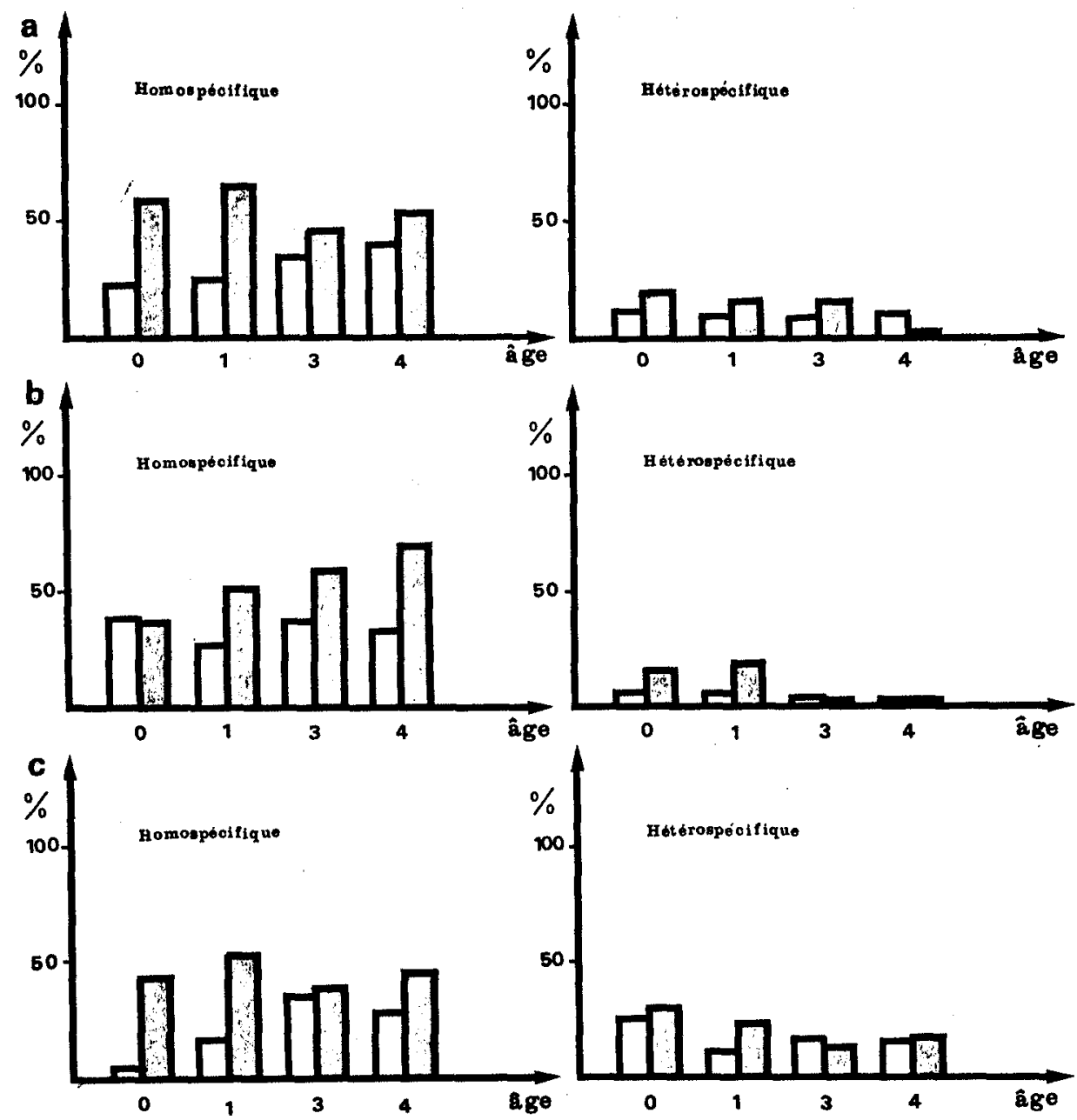

Fig. 2. - Nombre de comportements horno et hétérospécifiques sur le nombre total des comportements, en fonction de l'âge de mise en présence; $a$, trophallaxie; b, transport du couvain; $c$, toilette.

\section{$\square$ Camponotus senex.}

\section{Camponotus species.}

Fig. 2. - The number of homospecific and heterospecific behaviours of the total number of behaviours as a function of the age of the species when they are faced; a, trophallactic behaviour; b, brood-carrying; $c$, grooming. 
grande partie des activités hétérospécifiques et semblent donc «maintenir » les $C$. senex dans une situation que nous pouvons qualifier de légère dépendance (ou de profit).

\section{Camponotus senex - Pseudomyrmex ferruginea}

Cette association n'a pu être réalisée qu'avec des Pseudomyrmex âgés de moins de 3 heures après l'émergence. En effet, passé ce délai, leur comportement agressif est tel qu'ils ne tolèrent aucune autre espèce étrangère. La variation de l'âge de mise en présence n'affectera donc que les $C$. senex qui seront utilisées dès l'émergence ou à l'âge de 1 jour car, le $2^{\circ}$ jour, ces derniers exterminent très rapidement les $P$. ferruginea.

\section{Cohabitation}

Lorsque les $C$. senex sont mis en présence de $P$. ferruginea dès l'émergence, on ne remarque aucun conflit pour le partage de l'espace mais, lorsqu'ils sont âgés de 1 jour, les deux groupes ne fusionnent que le $2^{\circ}$ jour après la mise en présence (tableau I).

L'indice de tolérance sur la source de nourriture (tableau II) ne subit pratiquement pas de variations en fonction de l'âge de mise en présence des C. senex, celui-ci restant pratiquement constant : 0,68 ; par contre, il tend à augmenter en fonction de la durée de l'expérience.

La source de nourriture est plus fréquentée par les $C$. senex quand ils sont placés en colonie mixte à l'âge de 1 jour (81\%) que quand ils le sont dès l'émergence ( $41 \%$ ). Le phénomène inverse est observé chez $P$. ferruginea $\left(18,5 \%\right.$ contre $\left.58,5 \%, \chi^{2}=36,90, p<0,001\right)$.

Cependant, quand l'association se fait dès l'émergence, $C$. senex s'approvisionnent deux fois plus que dans les témoins tandis que chez $P$. ferruginea, ce comportement est moins fréquent que chez les témoins.

\section{Relations interindividuelles}

Les figures $3 a, b, c$ montrent que l'ensemble des activités homosipécifiques et hétérospécifiques sont assurées principalement par les $C$. senex.

Ainsi, dans l'association faite dès l'émergence, $C$. senex assure $39,5 \%$ des trophallaxies homospécifiques (contre $16,5 \%$ chez $P$. ferruginea), $\left(\chi^{2}=1,54\right.$, N.S.), et $42,5 \%$ des trophallaxies hétérospécifiques (contre $1,5 \%$ chez $P$. ferruginea). Alors que les échanges homospécifiques ont une durée moyenne ( $>30 \mathrm{~s}$ et $<60 \mathrm{~s}$ ) dans $95 \%$ des cas et courte $(<30 \mathrm{~s}$ ) dans $5 \%$, les échanges hétérospécifiques sont moyens à $73 \%$ et courts à $28 \%$. De même, les $C$. senex effectuent $50,5 \%$ du transport du couvain homospécifique (les $P$. ferruginea ne transportent que $15 \%$ de leur couvain) $-\chi^{2}=6,72, p<0,01$ ) - et $29 \%$ du transport hétérospécifique (les Pseudomyrmex n'ont jamais ce comportement).

Pour l'activité de toilette, la différence, moins nette, paraît aller dans 

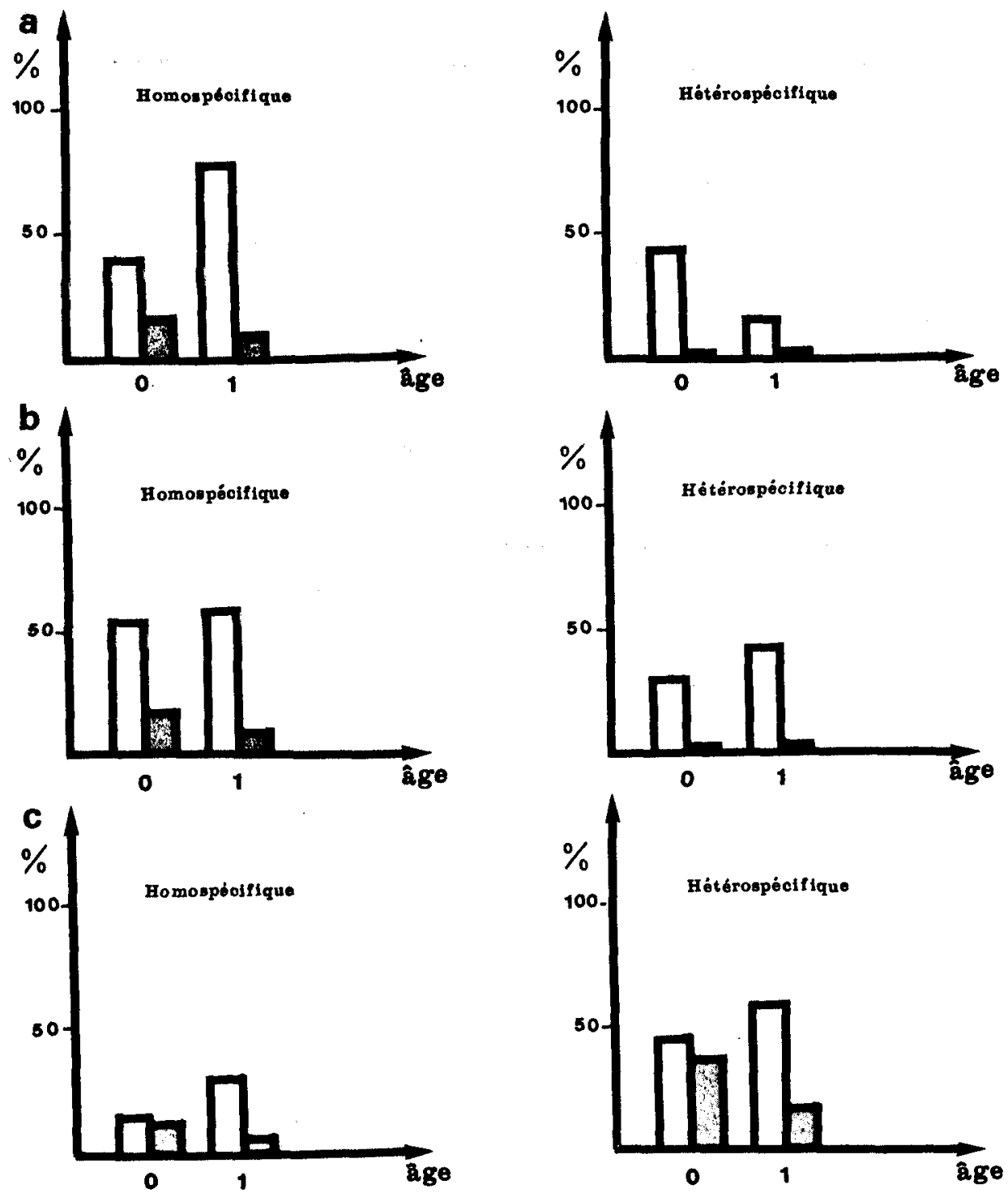

Fig. 3. - Nombre de comportements homo et hétérospécifiques sur le nombre total des comportements, en fonction de l'áge de mise en présence; $a$, trophallaxie ; $b$, transport du couvain; $c$, toilette.

\section{$\square$ Camponotus senex.}

- Pseudomyrmex ferruginea.

Fig. 3. - The number of homospecific and heterospecific behaviours of the total number of behaviours as a function of the age of the species when they are faced; $a$, trophallactic behaviour; $b$, brood-carrying; $c$, grooming. 
le même sens : $13,5 \%$ contre $11 \%$ pour les toilettes homospécifiques et $41 \%$ contre $34 \%$ pour les hétérospécifiques $\left(\chi^{2}\right.$, N.S.). Cette association met en évidence une relation du type parasitisme où l'essentiel des comportements actifs est assuré par les $C$. senex, les $P$. ferruginea n'ayant (pour les trophallaxies) qu'un rôle de receveuses et pratiquant même très souvent la cleptobiose.

\section{DISCUSSION}

Les expériences décrites ici montrent que les relations entre Camponotus senex et Camponotus abdominalis, Camponotus sp. ou Pseudomyrmex ferruginea, sont assez complexes.

Lorsque $C$. senex est associé à $C$. abdominalis, nous observons des relations prenant simultanément les caractères du parasitisme et de la symphilie, où $C$. senex est le principal bénéficiaire.

Dans l'association C. Senex -Camponotus sp. les relations semblent du même type, $C$. senex ayant un rôle souvent passif mais contribuant cependant à une partie des tâches de la colonie.

Enfin, dans l'association avec $P$. ferruginea, c'est au contraire $C$. senex qui serait $l$ 'hôte, $P$. ferruginea vivant à ses dépens et pratiquant même la "cleptobiose "lors des échanges homospécifiques entre Camponotus. Ces phénomènes sont très comparables à ceux observés dans la nature par Buschinger (1976), WHEELER (1921), WEBER (1943). Néanmoins, nous avons remarqué que $P$. ferruginea présentait fréquemment une activité domestique.

De plus, les comportements homospécifiques de $C$. senex évoluent selon le partenaire: leur proportion est très élevée dans l'association avec $P$. ferruginea tandis qu'elle reste faible en présence de $C$. abdominalis ou de Camponotus $\mathrm{sp}$. Les interactions entre ouvrières de $C$. senex dépendent donc aussi de la nature de l'espèce associée : dans l'association avec $P$. ferruginea, les $C$. senex sont obligés d'assurer la majorité des échanges interindividuels tandis qu'elles n'en effectuent qu'une partie dans les autres types d'associations, $C$. abdominalis et Camponotus sp. fournissant le complément.

En comparant la fréquence de ces comportements aux valeurs des colonies témoins n'associant qu'une espèce, on remarque que les $C$. abdominalis effectuent 2 fois plus d'échanges homospécifiques quand elles sont associées à $C$. senex, subissant ainsi une certaine stimulation de par cette présence. Par contre, ces comportements ne sont pas perturbés chez $C$. senex. De même, les Camponotus sp. présentent 5 fois plus de trophallaxie homospécifique et 3 fois plus de transport du couvain quand ils sont associés à $C$. senex, ces dernières étant également stimulées car elles effectuent 2 fois plus d'échanges que les animaux témoins. Enfin, lorsque $C$. senex cohabite avec $P$. ferruginea, les espèces subissent une forte stimulation, les trophallaxies homospécifiques étant 10 fois plus importantes que chez les témoins et les autres comportements 2 fois plus. 
La cohabitation s'établit d'autant plus tardivement que les fourmis sont rassemblées plus tard, ce phénomène serait dû essentiellement à l'âge des ouvrières lors de leur confrontation; étant donné que les fourmis des différentes colonies témoins sont toujours regroupées à proximité de l'abreuvoir, elles ont donc le même hygropreferendum quel que soit leur âge. Ceci rappelle donc un phénomène de type habituation ou du type "imprégnation", semblable à celui étudié dans la reconnaissance des cocons par les adultes (JAISSON, 1973, 1975; JAISSON et FRESNEAU, 1978). En effet, l'observation a montré que lorsque les individus sont rassemblés après un certain délai, une des espèces est souvent rejetée de la source de nourriture pendant une période plus ou moins longue. Ce comportement qui apparaît nettement dans les associations $C$. senex $-C$. abdominalis et $C$. senex $-P$. ferruginea rappelle les phénomènes de compétition trophique entre Camponotus et Crematogaster, Lasius et Myrmica, étudiées dans la nature par KaUDEWITZ (1955), DE VROEY (1979) et SwaIN (1980) et montre que la phase de tolérance serait d'autant plus grande que les espèces en présence disposent a priori d'un répertoire comportemental proche.

Dans le cas présent, cette hostilité s'atténuant plus ou moins rapidement, nous pouvons penser qu'il se forme, au sein de la colonie mixte, une odeur coloniale commune aux deux espèces. A moins que l'hostilité des deux partenaires soit réduite par suite d'une habituation rẻciproque et progressive, due à la contrainte du voisinage ayant débuté suffisamment tôt.

Ce problème fera l'objet de prochains travaux qui permettront peut-être d'expliquer les phénomènes de réactivité différentielle aux stimulations spécifiques intervenant dans les relations interindividuelles, notre but étant d'aller à l'encontre des mécanismes qui sous-tendent la mise en place des relations interspécifiques dans les sociétés mixtes naturelles, afin d'arriver à une meilleure compréhension de la division des rôles au sein de ces colonies.

\section{Bibliographie}

BenoIs A., 1969. - Etude morphologique, biologique et éthologique de Camponotus vagus scop. Thèse Doct. $3^{\circ}$ cycle, Toulouse, $280 \mathrm{p}$.

BUsChINGER A., 1976. - Eine Methode zur Zucht der Gastameise Formicoxenus nitidulus (Nyl.) mit Leptothorax acevorum (Fabr.) als "Wirtsameise" (Hym., Form.). Ins. Soc., 23, 205-214.

DE VROEY C., 1979. - Relations interspécifiques chez les fourmis. C.R. Sct. Fr. U.I.E.I.S., Lausanne, 107-113.

Fielde A.M., 1903. - Artificial mixed nests of ants. Biol. Bull., 5, 320-325.

FOREL A., 1898. - La parabiose chez les fourmis. Bull. Soc. Vaud. Sc. Nat., 34, 380-384.

HEYDE H.C., 1924. - Die Entwicklung der psychichen Fähigkeiten bei Ameisen und ihr Verhalten bei abgeänderten biologischen Bedingungen. Biol. Zentralbl., 44, 623-654.

JAISsoN P., 1973. - L'imprégnation dans l'ontogenèse du comportement de soins aux cocons chez les Formicines. Proc. VIIth Congr. I.U.S.S.I., Londres, 176-181.

Jarsson P., 1975. - L'imprégnation dans l'ontogenèse des comportements de soins aux cocons chez la jeune fourmi rousse (Formica polyctena Först.). Behaviour, 52, 1-37.

JaIsson P., 1980. - Les colonies mixtes plurispécifiques: un modèle pour l'étude des fourmis. Bull. Ecol. Médit., VII, 163-166. 
JaIsson P., Fresneau D., 1978. - The sensitivity and responsiveness of ants to their cocoons in relation to age and methods of measurement. Animal Behaviour, 26, 1064-1071.

KAUDEWITZ F., 1955. - Zum Gastverhältnis zwischen Crematogaster scutellaris Ol. mit Camponotus lateralis bicolor Ol. Biol. Zentr. Blatt., 74, 69-87.

KutreR H., 1945. - Eine neue Ameisengattung Doronomyrmex. Bull. Soc. Entomol, Suisse, $19,485-487$.

ManN W.M., 1912. - Parabiosis in Brazilian ants. Psyche, Cambridge, 19, 36-41.

Plateaux L., 1960 a. - Adoptions expérimentales de larves entre des fourmis de genre différent: (III) Anergates atratulus Schenck et Solenopsis fugax Latreille; (IV) Leptothorax nylanderi Förster et Tetramorium caespitum L. Ins. Soc., 7, 345.348.

Plateaux L., 1980 b. - Adoptions expérimentales de larves entre des fourmís de genre différent: Leptothorax nylanderi Förster et Solenopsis fugax Latreille. Ins. Soc., 7. 163-170.

StUMPER R., 1950. - Etudes myrmécologiques. X: La myrmécobiose. Bull. Soc. Nat. Luxemb., 44, 31-43.

SwaIN R.B., 1980. - Trophic competition among parabiotic ants. Ins. Soc., Paris, 27, 377-390.

TAYLOR R.W., 1978. - Nothomyrmecia macrops: a living fossil ant rediscovered. Science, 201, 979-985.

WhEELER W.M., 1921. - A new case of parabiosis and the "ant gardens" of British Guiana. Ecology, 2, 89-103.

WheEler W.M., 1926. - Les sociétés d'insectes, Doin, Paris.

Weber N.A., 1943. - Parabiosis in neotropical " ant gardens". Ecology, 24, 400-404. 